\title{
Cost of prenatal versus postnatal myelomeningocele closure for both mother and child at 1 year of life
}

\author{
Brice A. Kessler, MD, ${ }^{1}$ Michael P. Catalino, MD, MS, ${ }^{1}$ Carolyn Quinsey, MD, ${ }^{1}$ \\ William Goodnight, MD, MSCR, ${ }^{2}$ and Scott Elton, MD'
}

\begin{abstract}
Departments of ${ }^{1}$ Neurosurgery and ${ }^{2}$ Obstetrics \& Gynecology, University of North Carolina School of Medicine, Chapel Hill, North Carolina
\end{abstract}

\begin{abstract}
OBJECTIVE Prenatal myelomeningocele (MMC) closure has been performed in the United States for 2 decades. While prior work has focused on clinical outcomes of prenatal MMC closure, the cost of this procedure in comparison with that of postnatal MMC closure is unclear. The authors' aim was to compare the cost of prenatal versus postnatal MMC closure for both the child and mother at 1 year.
\end{abstract}

METHODS A prospective database of patients undergoing prenatal and postnatal MMC closure between 2011 and 2018 with 1-year follow-up was retrospectively reviewed. Charge data for relevant admissions were converted to a cost estimate using the authors' institution's Medicare hospital-specific cost-to-charge ratio. Children, mothers, and mother/ child pairs were considered separately. The primary outcome was cost. Secondary outcomes included the need for hydrocephalus treatment, length of stay (LOS), and readmissions. Other covariates included gestational age at birth, MMC lesion level, and obstetric complications.

RESULTS The median cost of care for children in the prenatal group was greater, although not significantly so, at $\$ 58,406.71$ (IQR \$16,900.24-\$88,951.01) compared with \$49,889.95 (IQR \$38,425.18-\$115,163.86) for children in the postnatal group $(p=0.204)$. The median cost for mothers in the prenatal group was significantly greater at $\$ 24,548.29$ (IQR \$20,231.55-\$36,862.31) compared with \$5087.30 (IQR \$4430.72-\$5362.56) ( $<<0.001)$. The median cost for mother/child pairs in the prenatal group was $\$ 102,377.75$ (IQR \$37,384.30-\$118,527.74) compared with \$55,667.82 (IQR $\$ 42,840.78-\$ 120,058.06)(p=0.45)$. Children in the prenatal group had a lower gestational age at birth $(235.81$ days vs 265.77 days, $p<0.001$ ) and fewer readmissions (33.3\% vs $72.7 \%, p<0.001)$, and hydrocephalus treatment was less common (33.3\% vs $90.9 \%, p<0.001)$. Index LOS did not differ between children in the prenatal and postnatal groups (26.8 days vs 23.5 days, $p=0.63$ ). Mothers in the prenatal group had longer LOS ( 15.92 days vs 4.68 days, $p<0.001)$ and more readmissions ( $18.5 \%$ vs $0.0 \%, p=0.06)$.

CONCLUSIONS The median cost of prenatal versus postnatal MMC closure did not significantly differ from a hospital perspective at 1 year, although variability in cost was high for both groups. When considering the mother alone, prenatal MMC closure was costlier. Future work is needed to assess cost from a patient and societal perspective both at 1 year and beyond.

https://thejns.org/doi/abs/10.3171/2019.7.FOCUS19417

KEYWORDS myelomeningocele; prenatal surgery; intrauterine surgery; cost; hydrocephalus

$\mathrm{M}$ YELOMENINGOCELE (MMC) is characterized by protrusion of neural elements through an unfused portion of the spinal column in utero. ${ }^{1,10}$ While the incidence of MMC has decreased in the United States in part due to folic acid supplementation, ${ }^{19}$ the lifetime care of patients born with MMC remains costly. The cost of care estimates for patients with MMC are 13 times greater in childhood and 3-6 times costlier in adulthood than for individuals without MMC. ${ }^{6,13}$ This cost is driven by the lifelong clinical sequelae of MMC including hydrocephalus, bowel and bladder dysfunction, and lower-extremity paresis/paralysis. ${ }^{2-4,8,12,13}$ Additionally, the need for hydrocephalus treatment with ventriculoperitoneal shunt placement or endoscopic third ventriculostomy (ETV)

ABBREVIATIONS CCR = cost-to-charge ratio; $\mathrm{CPC}=$ choroid plexus cauterization; $\mathrm{ETV}=$ endoscopic third ventriculostomy; LOS = length of stay; $\mathrm{MMC}=$ myelomeningocele; $\mathrm{MOMS}=$ Management of Myelomeningocele Study; PPROM = preterm premature rupture of membranes.

SUBMITTED May 28, 2019. ACCEPTED July 16, 2019.

INCLUDE WHEN CITING DOI: 10.3171/2019.7.FOCUS19417. 
with or without choroid plexus cauterization (CPC) is high in MMC patients, putting them at risk of infectious and mechanical failures related to these procedures. ${ }^{2,4,8,9}$

Prenatal, or intrauterine, MMC closure has been performed in the United States for over 2 decades, whereas historically MMC defects were closed postnatally. ${ }^{1}$ Utilization of prenatal MMC closure has increased since the landmark Management of Myelomeningocele Study (MOMS) trial demonstrated a reduced need for ventricular shunting for treatment of hydrocephalus and improved motor outcomes. ${ }^{1,5,7,10}$ However, prenatal MMC closure is not without risks to the fetus, including oligohydramnios, preterm delivery, and perinatal death. . $, 5,7,10$ Obstetric risks to the mother must also be considered, including initial perioperative risks, premature rupture of membranes, and risks to future pregnancies. ${ }^{1,10,11,16}$ Prenatal MMC closure requires significant upfront utilization of hospital resources compared with postnatal MMC closure, and nearly all of this care is provided in the hospital setting, given the complexity of these procedures.

To our knowledge, data on comparative hospital costs for prenatal versus postnatal MMC closure are limited. The goal of this study was to compare the 1-year hospital costs associated with prenatal and postnatal MMC closure for both the child and mother. Given the demonstrated benefit of reduced hydrocephalus treatment with prenatal MMC closure, our hypothesis was that the costs in the prenatal group would be less than or equal to those in the postnatal group.

\section{Methods}

All patients undergoing prenatal MMC closure at our institution between 2011 and 2018 with 1 year of followup were included. Children and mothers in the prenatal group were identified through a prospective departmental database and retrospectively reviewed. Children and mothers in the postnatal group were identified by querying our institution's data warehouse for Current Procedural Terminology (CPT) codes related to MMC repair (CPT 63706 and 63074). This study was approved by the institutional review board. We considered mother and child as part of a pair and followed both from surgery through the end of the 1 st year of the child's life. If patients were lost to follow-up during that time period, they were excluded.

The primary outcome was hospital inpatient cost. Hospital inpatient charge data were obtained for relevant admissions. Charges falling under dates of unrelated admissions were excluded. Cost was estimated by obtaining our institution's Medicare hospital-specific cost-tocharge ratio (CCR) for each relevant year (https://www. cms.gov/Medicare/Medicare-Fee-for-Service-Payment/ AcuteInpatientPPS/Historical-Impact-Files-for-FY1994-through-Present.html). Total charges were multiplied by the CCR to estimate cost. ${ }^{15}$ Costs were adjusted to 2018 US dollars with the United States Department of Labor Consumer Price Index inflation calculator (https:// www.bls.gov/data/inflation_calculator.htm).

Secondary outcomes were the need for hydrocephalus treatment (shunting or ETV/CPC) within the 1st year, length of stay (LOS), gestational age at birth, and 1-year readmission rates. We obtained LOS data including in- dex LOS for MMC closure children, index LOS for initial surgery for mothers in the prenatal group, and index LOS from delivery for all mothers. Readmission within 1 year and associated LOS for readmissions were recorded. As prenatal MMC closure reduces the subsequent need for treatment of hydrocephalus, readmissions directly related to hydrocephalus treatment were recorded. All-cause readmissions were also recorded. Other covariates included whether the upper level of the lesion was at or above the L2 vertebra versus L3 vertebra and below, the need for wound revision, and obstetric complications including oligohydramnios (amniotic fluid index $<5 \mathrm{~cm}$ on ultrasound), preterm premature rupture of membranes (PPROM, defined and as any spontaneous rupture on or before 34 weeks 0 days), and preterm delivery at 34 weeks 0 days or earlier. Categorical variables are presented as proportions and comparisons made using Fisher's exact test. Parametric continuous variables are presented as means, and comparisons were made using a 2-tailed t-test. Nonparametric continuous variables are presented as medians and IQR, and comparisons were made using the Mann-Whitney U-test.

\section{Results}

We identified 38 prenatal and 32 postnatal MMC closure procedures performed between 2011 and 2018. In the prenatal group, 5 mother/child pairs were excluded as portions of care took place elsewhere and 6 pairs were excluded due to less than 1 year of follow-up data. Of the 27 pairs included in the prenatal MMC group, 2 had unsuccessful MMC closures, and the procedures were aborted with subsequent successful postnatal closure. These 2 unsuccessful prenatal MMC closures were included in the prenatal group. Eight pairs within the postnatal group were excluded because the mother gave birth elsewhere, and 2 pairs were excluded due to less than 1 year of follow-up. A total of 22 pairs were included in the postnatal group.

Of the 22 postnatal mothers included, 20 underwent screening for prenatal MMC closure. Five of these were otherwise qualified for prenatal MMC closure but chose postnatal MMC closure after being counseled of the risks and benefits in clinic. Fifteen of the 20 patients who were screened did not qualify for prenatal MMC closure based on the criteria put forth by the MOMS trial; 3 mothers had a BMI $>35 \mathrm{~kg} / \mathrm{m}^{2}, 4$ had secondary fetal anomalies on ultrasound, and 8 presented to the clinic with advanced gestational age ( $>26$ weeks). The 2 mothers who were not screened presented to our institution on the day of birth.

The median cost of care for children in the prenatal group was greater, although not significantly so, at $\$ 58,406.71$ (IQR $\$ 16,900.24-\$ 88,951.01$ ) compared with $\$ 49,889.95$ (IQR $\$ 38,425.18-\$ 115,163.86$ ) for children in the postnatal group $(\mathrm{p}=0.204)$. The median cost for mothers in the prenatal group was significantly greater at \$24,548.29 (IQR \$20,231.55-\$36,862.31) compared with $\$ 5087.30$ (IQR \$4430.72-\$5362.56) ( $p<0.001)$. The median cost for mother/child pairs in the prenatal group was $\$ 102,377.75$ (IQR $\$ 37,384.30-\$ 118,527.74$ ) compared with $\$ 55,667.82$ (IQR $\$ 42,840.78-\$ 120,058.06)(\mathrm{p}=0.45)$ (Fig. 1).

Comparative patient clinical characteristics as well as cost and outcome data are presented in Table 1. The aver- 
MMC Mothers

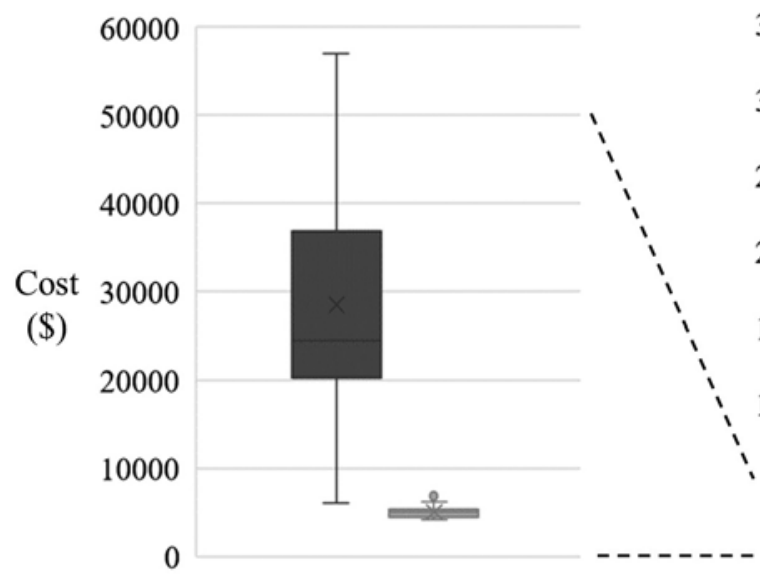

Prenatal MMC Closure

Postnatal MMC Closure
MMC Children

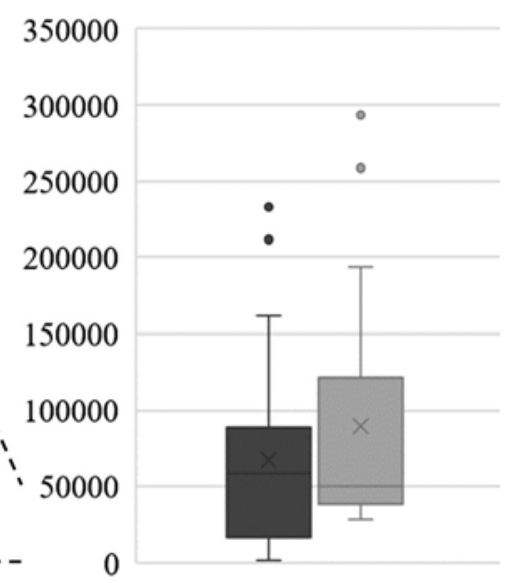

MMC Mother/Child Pair

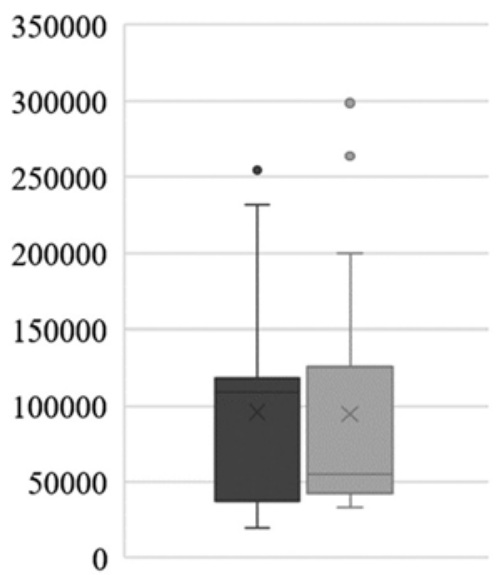

FIG. 1. Cost comparison of prenatal versus postnatal MMC closure for mothers, children, and mother/child pair. Results are presented as box-and-whisker graphs with dots representing outliers, the line within the box representing the median, and the $X$ representing the mean.

age gestational age at time of surgery for children in the prenatal group was 171.3 days ( 24.5 weeks). Children in the prenatal group had lower gestational ages at birth (235.8 days [33.6 weeks] vs 265.8 days [38.0 weeks], p < 0.001). Index LOS from birth to discharge did not differ between children in the prenatal and postnatal groups (26.8 days vs 23.5 days, $p=0.631$ ). Similarly, total inpatient LOS for children in the prenatal versus postnatal groups including readmission LOS did not differ at 1 year (27.8 days vs 25.7 days, $\mathrm{p}=0.781$ ). Readmissions related to hydrocephalus were less common for children in the prenatal group $(33.3 \%$ vs $72.7 \%$, $\mathrm{p}<0.001)$. All-cause readmission was less common for children in the prenatal group $(41.1 \%$ vs $81.8 \%, \mathrm{p}<0.019)$. Additionally, the average number of all-cause readmissions was less for prenatal MMC closure children (0.56 vs 1.68, p = 0.001). For prenatal MMC closure children, causes for readmission aside from treatment of hydrocephalus included urinary tract infection $(n=1)$, fever of unknown origin $(n=2)$, and gastroenteritis $(n=$ 2). For postnatal MMC closure children causes for readmission aside from treatment of hydrocephalus included upper respiratory infection $(n=5)$, apnea $(n=2)$, urinary tract infection $(n=2)$, feeding difficulties $(n=1)$, gastroenteritis $(n=1)$, fever of unknown origin $(n=1)$, and seizures $(n=1)$. Treatment of hydrocephalus at 1 year was less common for children in the prenatal group $(29.6 \%$ vs $90.9 \%, \mathrm{p}<0.001)$. Of all children who required subsequent treatment for hydrocephalus, there was no difference in the proportion of children undergoing hydrocephalus treatment during the index hospital stay or subsequently during readmission between prenatal and postnatal groups $(25.0 \%$ vs $45.0 \%, p=0.419)$. Of the 8 children requiring hydrocephalus treatment in the prenatal group, 5 received ventriculoperitoneal shunts, 2 underwent ETV/CPC, and 1 underwent ETV alone. Of the 20 children in the postnatal group who underwent hydrocephalus treatment, 19 received a ventriculoperitoneal shunt, and 1 underwent ETV.
No children from either group required MMC wound revisions. The proportion of children with lower MMC lesion levels (L3 or lower) did not differ between groups $(77.8 \%$ vs $72.7 \%, p=0.747)$.

The index LOS, or LOS related to MMC closure, for mothers in the prenatal group was 5.41 days. The LOS for delivery was significantly longer for mothers in the prenatal group (10.65 days vs 4.68 days, $\mathrm{p}=0.037$ ). Only 1 mother in the postnatal group had a preterm delivery defined as less than 34 weeks' gestation due to eclampsia and hemolysis, elevated liver enzyme levels, and low platelet levels (HELLP syndrome), compared with $63 \%$ of mothers in the prenatal group delivering before 34 weeks $(\mathrm{p}<$ 0.001 ). PPROM and oligohydramnios occurred in $51.9 \%$ and $18.5 \%$ of prenatal mothers, respectively. Prepartum data for postnatal repair mothers was not consistently available. No readmissions occurred for mothers in the postnatal group, while $18.5 \%$ of prenatal MMC closure mothers were readmitted ( $\mathrm{p}=0.056)$. This included readmissions between MMC closure and delivery $(n=4)$, and subsequent to delivery $(n=1)$. The total inpatient LOS at 1 year was greater for mothers in the prenatal group (15.9 days vs 4.7 days, $\mathrm{p}<0.001)$.

\section{Discussion}

The median cost of prenatal versus postnatal MMC closure did not significantly differ when comparing children or mother/child pairs in both groups. Cost for both groups of children had significant variability, with costly outliers giving the data a positive skew (Fig. 1). These costs were overall greater for children in the postnatal group (IQR $\$ 38,425.18-\$ 115,163.86$ vs IQR \$16,900.24-\$88,951.01), which may be due to the higher rates of readmission and hydrocephalus in children who undergo postnatal MMC closure (Table 1). This may also reflect the number of mothers in the postnatal closure group who were initially 
TABLE 1. Clinical characteristics and cost associated with prenatal versus postnatal MMC closure for children, mothers, and mother/child pairs at 1 year of life

\begin{tabular}{|c|c|c|c|}
\hline & Prenatal MMC Closure $(n=27)$ & Postnatal MMC Closure $(n=22)$ & p Value \\
\hline \multicolumn{4}{|l|}{ Child } \\
\hline Average GA at MMC closure (days) & 171.3 & NA & \\
\hline Average GA at birth (days) & 235.8 & 265.8 & $<0.001$ \\
\hline Lesion level L3 or below & $77.8 \%(21)$ & $72.7 \%(16)$ & 0.747 \\
\hline Average index LOS (days) & 26.8 & 23.5 & 0.631 \\
\hline Average total inpatient LOS (days) & 27.8 & 25.7 & 0.781 \\
\hline All-cause readmission & $41.1 \%(13)$ & $81.8 \%(18)$ & 0.019 \\
\hline Average no. of all-cause readmissions & 0.56 & 1.68 & 0.001 \\
\hline Hydrocephalus readmissions & $33 \%(9)$ & $72.7 \%(16)$ & $<0.001$ \\
\hline CSF diversion required & $29.6 \%(8)$ & $90.9 \%(20)$ & $<0.001$ \\
\hline During index hospital stay & $25.0 \%(2 / 8)$ & $45.0 \%(9 / 20)$ & 0.419 \\
\hline Median inpatient cost $(\$)$ & $58,406.71$ & $49,889.95$ & 0.204 \\
\hline \multicolumn{4}{|l|}{ Mother } \\
\hline Average LOS at MMC closure (days) & 5.41 & NA & \\
\hline Average delivery LOS (days) & 10.7 & 4.7 & 0.037 \\
\hline Average total inpatient LOS (days) & 15.9 & 4.7 & $<0.001$ \\
\hline Preterm delivery & $63.0 \%(17)$ & $4.5 \%(1)$ & $<0.001$ \\
\hline PPROM & $51.9 \%(14)$ & - & \\
\hline Oligohydramnios & $18.5 \%(5)$ & - & \\
\hline Obstetric readmission & $18.5 \%(5)$ & $0.0 \%(0)$ & 0.056 \\
\hline Median inpatient cost (\$) & $24,548.29$ & $5,087.30$ & $<0.001$ \\
\hline \multicolumn{4}{|l|}{ Mother \& child } \\
\hline Median total inpatient cost (\$) & $102,377.75$ & $55,667.82$ & 0.451 \\
\hline
\end{tabular}

$\mathrm{GA}=$ gestational age; $\mathrm{NA}=$ not applicable $;-=$ prepartum care data not consistently available for the mothers in the postnatal group.

screened for prenatal MMC closure and did not qualify due to secondary fetal anomalies and high maternal BMI, as these may have negatively impacted the child's health and increased use of healthcare resources after birth. However, our small cohort numbers may have limited our ability to detect a statistical significance in the setting of such variability.

For mothers in the prenatal closure group, the cost was more than 5 times that of mothers in the postnatal group. This is expected, as prenatal MMC closure requires 2 separate surgical procedures and inpatient stays. Additionally, $18.5 \%$ of mothers in the prenatal group experienced a readmission for an obstetric complication, while no mothers in the postnatal group were readmitted. Even the delivery LOS was longer for mothers in the prenatal group than for those in the postnatal group. This is possibly secondary to admission for management of an obstetric complication after prenatal MMC closure but prior to planned delivery, with unplanned delivery of the child during that admission. Interestingly, the IQRs for prenatal and postnatal MMC mother/child pairs are similar $(\$ 37,384.30-\$ 118,527.74$ vs $\$ 42,840.78-\$ 120,058.06)$. While the addition of the mother's cost for a postnatal repair added relatively little to the overall cost of the postnatal MMC mother/child pair, the mother's cost for a prenatal MMC closure was significantly greater than that for postnatal MMC mothers, increasing the overall cost of prenatal MMC mother/child pairs a great deal. Even with this addition, there was no significant difference in the median cost between the mother/child pairs. This again may reflect the high variability of the children's cost and our smaller study numbers.

Despite no statistical difference in cost, prenatal MMC closure children were less likely to be readmitted within 1 year and less likely to undergo shunt placement. This is in line with previous reports demonstrating reduction in rates of hydrocephalus with prenatal MMC closure. ${ }^{1,10,17}$ Hydrocephalus and shunt management is costly, ${ }^{14}$ and this is a cost that follows MMC patients throughout their life. ${ }^{8,13}$ As prenatal MMC closure children undergo shunt placement less frequently, they are spared from the known risk of mechanical and infectious complications of shunt placement. ${ }^{2,9}$ While we did not detect a difference in cost between children who underwent prenatal and postnatal closure at 1 year, it is possible that the lifelong cost is reduced without the need for ongoing treatment of complications related to hydrocephalus. Longer follow-up will be critical to estimate the economic impact of lower hydrocephalus and readmission rates in children.

A cost-effectiveness model of prenatal MMC closure was reported by Werner et al. based largely on data from the MOMS as well as published probability and cost data. ${ }^{18}$ This model demonstrated cost-effectiveness of prenatal MMC closure but was restricted to the subpopulation of pregnancies included within the MOMS trial. We at- 
tempted here to generate primary cost data based on our cohort of MMC patients, with patients in both prenatal and postnatal groups who may not have qualified for prenatal MMC closure based solely on MOMS trial criteria. Cost was estimated by multiplying charge data by our hospitalspecific Medicare CCR, which is an aggregate CCR for 1 year at our hospital. Costs calculated via this method are likely a reasonable estimate of direct costs to the hospital when comparing groups of patients within that hospital, but this method may have limited generalizability. ${ }^{15}$ This method also estimates cost via a hospital-specific CCR and reflects cost to the hospital, not to the patient or society. We estimated cost at 1 year of life in our study based on the time period reported by the original MOMS trial. ${ }^{1}$ Certainly, ramifications of prenatal versus postnatal MMC closure extend beyond 1 year. Further work will be necessary to assess if over a lifetime the cost of prenatal MMC closure and its clinical sequelae outweighs or is less than the cost associated with postnatal MMC closure.

\section{Conclusions}

The median cost of prenatal versus postnatal MMC closure, including costs associated with both mother and child, did not significantly differ at our institution from a hospital perspective at 1 year. Variability of cost was high for children in both groups. Prenatal MMC closure was costlier when considering care of the mother. Readmissions and treatment for hydrocephalus were more common for children in the postnatal group. It is possible that beyond 1 year of life, comparative cost may be greater for postnatal MMC closure children given their higher readmission rate and need for subsequent hydrocephalus management, but a difference in cost from a hospital perspective was not seen in our patient cohorts at 1 year. Future work is needed to assess costs from a patient and societal perspective both at 1 year and beyond.

\section{References}

1. Adzick NS, Thom EA, Spong CY, Brock JW III, Burrows PK, Johnson MP, et al: A randomized trial of prenatal versus postnatal repair of myelomeningocele. $\mathbf{N}$ Engl J Med 364:993-1004, 2011

2. Berry JG, Hall MA, Sharma V, Goumnerova L, Slonim AD, Shah SS: A multi-institutional, 5-year analysis of initial and multiple ventricular shunt revisions in children. Neurosurgery 62:445-454, 2008

3. Brock JW III, Carr MC, Adzick NS, Burrows PK, Thomas JC, Thom EA, et al: Bladder function after fetal surgery for myelomeningocele. Pediatrics 136:e906-e913, 2015

4. Caldarelli M, Di Rocco C, La Marca F: Shunt complications in the first postoperative year in children with meningomyelocele. Childs Nerv Syst 12:748-754, 1996

5. Cavalheiro S, da Costa MDS, Moron AF, Leonard J: Comparison of prenatal and postnatal management of patients with myelomeningocele. Neurosurg Clin N Am 28:439448, 2017

6. Centers for Disease Control: Economic burden of spina bifida-United States, 1980-1990. Clin Pediatr 29:198-199, 1990

7. Cherian J, Staggers KA, Pan IW, Lopresti M, Jea A, Lam S: Thirty-day outcomes after postnatal myelomeningocele repair: a National Surgical Quality Improvement Program Pe- diatric database analysis. J Neurosurg Pediatr 18:416-422, 2016

8. Davis BE, Daley CM, Shurtleff DB, Duguay S, Seidel K, Loeser JD, et al: Long-term survival of individuals with myelomeningocele. Pediatr Neurosurg 41:186-191, 2005

9. Dupepe EB, Hopson B, Johnston JM, Rozzelle CJ, Jerry Oakes W, Blount JP, et al: Rate of shunt revision as a function of age in patients with shunted hydrocephalus due to myelomeningocele. Neurosurg Focus 41(5):E6, 2016

10. Farmer DL, Thom EA, Brock JW III, Burrows PK, Johnson MP, Howell LJ, et al: The Management of Myelomeningocele Study: full cohort 30-month pediatric outcomes. Am J Obstet Gynecol 218:256.e1-256.e13, 2018

11. Johnson MP, Bennett KA, Rand L, Burrows PK, Thom EA, Howell LJ, et al: The Management of Myelomeningocele Study: obstetrical outcomes and risk factors for obstetrical complications following prenatal surgery. Am J Obstet Gynecol 215:778.e1-778.e9, 2016

12. Mukherjee S, Pasulka J: Care for adults with spina bifida: current state and future directions. Top Spinal Cord Inj Rehabil 23:155-167, 2017

13. Ouyang L, Grosse SD, Armour BS, Waitzman NJ: Health care expenditures of children and adults with spina bifida in a privately insured U.S. population. Birth Defects Res A Clin Mol Teratol 79:552-558, 2007

14. Patwardhan RV, Nanda A: Implanted ventricular shunts in the United States: the billion-dollar-a-year cost of hydrocephalus treatment. Neurosurgery 56:139-145, 2005

15. Shwartz M, Young DW, Siegrist R: The ratio of costs to charges: how good a basis for estimating costs? Inquiry 32:476-481, 1995-1996

16. Soni S, Moldenhauer JS, Spinner SS, Rendon N, Khalek N, Martinez-Poyer J, et al: Chorioamniotic membrane separation and preterm premature rupture of membranes complicating in utero myelomeningocele repair. Am J Obstet Gynecol 214:647.e1-647.e7, 2016

17. Tulipan N, Wellons JC III, Thom EA, Gupta N, Sutton LN, Burrows PK, et al: Prenatal surgery for myelomeningocele and the need for cerebrospinal fluid shunt placement. J Neurosurg Pediatr 16:613-620, 2015

18. Werner EF, Han CS, Burd I, Lipkind HS, Copel JA, Bahtiyar $\mathrm{MO}$, et al: Evaluating the cost-effectiveness of prenatal surgery for myelomeningocele: a decision analysis. Ultrasound Obstet Gynecol 40:158-164, 2012

19. Yen IH, Khoury MJ, Erickson JD, James LM, Waters GD, Berry RJ: The changing epidemiology of neural tube defects. United States, 1968-1989. Am J Dis Child 146:857-861, 1992

\section{Disclosures}

The authors report no conflict of interest concerning the materials or methods used in this study or the findings specified in this paper.

\section{Author Contributions}

Conception and design: Elton, Kessler. Acquisition of data: Kessler. Analysis and interpretation of data: Elton, Kessler, Catalino, Quinsey. Drafting the article: Elton, Kessler, Catalino, Quinsey. Critically revising the article: Elton, Catalino, Quinsey, Goodnight. Reviewed submitted version of manuscript: all authors. Statistical analysis: Catalino. Study supervision: Elton.

\section{Correspondence}

Scott Elton: University of North Carolina School of Medicine, Chapel Hill, NC. scott_elton@med.unc.edu. 